\title{
THE DETERMINANT BOUND FOR DISCREPANCY IS ALMOST TIGHT
}

\author{
JIŘÍ MATOUŠEK
}

(Communicated by Jim Haglund)

\begin{abstract}
In 1986 Lovász, Spencer, and Vesztergombi proved a lower bound for the hereditary discrepancy of a set system $\mathcal{F}$ in terms of determinants of square submatrices of the incidence matrix of $\mathcal{F}$. As shown by an example of Hoffman, this bound can differ from herdisc $(\mathcal{F})$ by a multiplicative factor of order almost $\log n$, where $n$ is the size of the ground set of $\mathcal{F}$. We prove that it never differs by more than $O\left((\log n)^{3 / 2}\right)$, assuming $|\mathcal{F}|$ bounded by a polynomial in $n$. We also prove that if such an $\mathcal{F}$ is the union of $t$ systems $\mathcal{F}_{1}, \ldots, \mathcal{F}_{t}$, each of hereditary discrepancy at most $D$, then $\operatorname{herdisc}(\mathcal{F}) \leq O\left(\sqrt{t}(\log n)^{3 / 2} D\right)$. For $t=2$, this almost answers a question of Sós. The proof is based on a recent algorithmic result of Bansal, which computes low-discrepancy colorings using semidefinite programming.
\end{abstract}

\section{INTRODUCTION}

Let $V=[n]:=\{1,2, \ldots, n\}$ be a vertex set and $\mathcal{F}=\left\{F_{1}, F_{2}, \ldots, F_{m}\right\}$ be a system of subsets of $V$. The discrepancy of $\mathcal{F}$ is $\operatorname{disc}(\mathcal{F}):=\min _{\chi} \operatorname{disc}(\mathcal{F}, \chi)$, where the minimum is over all colorings $\chi: V \rightarrow\{-1,+1\}$, and $\operatorname{disc}(\mathcal{F}, \chi):=$ $\max _{i=1,2, \ldots, m}\left|\sum_{j \in F_{i}} \chi(j)\right|$.

The hereditary discrepancy of $\mathcal{F}$ is

$$
\operatorname{herdisc}(\mathcal{F}):=\max _{J \subseteq V} \operatorname{disc}\left(\left.\mathcal{F}\right|_{J}\right) .
$$

Here $\left.\mathcal{F}\right|_{J}$ denotes the restriction of the set system $\mathcal{F}$ to the ground set $J$, i.e., $\{F \cap J: F \in \mathcal{F}\}$.

Bounding the discrepancy or the hereditary discrepancy of a particular set system from below is usually challenging. One of the strongest known tools is a result known as the determinant lower bound. To formulate it we define, for a real matrix $A$,

$$
\operatorname{detlb}(A):=\max _{k} \max _{B}|\operatorname{det} B|^{1 / k},
$$

where the maximum is over all $k \times k$ submatrices $B$ of $A$. For a set system $\mathcal{F}$, we put $\operatorname{detlb}(\mathcal{F}):=\operatorname{detlb}(A)$, where $A$ is the incidence matrix of $\mathcal{F}$.

Theorem 1 (Lovász, Spencer, and Vesztergombi [LSV86]). For every (finite) set system $\mathcal{F}$ we hav $11 \operatorname{herdisc}(\mathcal{F}) \geq \frac{1}{2} \operatorname{detlb}(\mathcal{F})$.

Received by the editors January 4, 2011 and, in revised form, July 2, 2011.

2010 Mathematics Subject Classification. Primary 05D99.

The author was partially supported by the ERC Advanced Grant No. 267165.

${ }^{1}$ The bound in [LSV86] is stated without the $\frac{1}{2}$ factor. This has two causes: first, their discrepancy is scaled by $\frac{1}{2}$ compared to ours, and second, in their argument, at one step they seem to be multiplying by 2 whereas, in my opinion, one should divide by 2 . 
Lovász et al. LSV86] conjectured that the determinant lower bound is tight up to a constant factor, i.e., $\operatorname{herdisc}(\mathcal{F})=O(\operatorname{detlb}(\mathcal{F}))$ for all $\mathcal{F}$. This was refuted by an example of Hoffmann 2 which shows that herdisc $(\mathcal{F}) / \operatorname{detlb}(\mathcal{F})$ can be of order $(\log n) /(\log \log n)$. A construction of Pálvölgyi Pál10, also presented in Section 5 below, provides the slightly stronger lower bound of $\Omega(\log n)$ for the same quantity.

Here we prove that herdisc $(\mathcal{F}) / \operatorname{detlb}(\mathcal{F})$ cannot be much larger than in these examples, at least if $|\mathcal{F}|$ is bounded by a polynomial function of $n$.

Theorem 2. For every set system $\mathcal{F}$,

$$
\operatorname{herdisc}(\mathcal{F}) \leq \operatorname{detlb}(\mathcal{F}) \cdot O(\log (m n) \sqrt{\log n}) .
$$

Next, we consider the situation where a set system $\mathcal{F}$ as above is a union of set systems $\mathcal{F}_{1}, \mathcal{F}_{2}, \ldots, \mathcal{F}_{t}$, g and we are interested in bounding $\operatorname{herdisc}(\mathcal{F})$ in terms of the hereditary discrepancies of the $\mathcal{F}_{i}$.

For $t=2$, this problem was raised by Sós (it is cited, e.g., in [LSV86]). She asked whether herdisc $\left(\mathcal{F}_{1} \cup \mathcal{F}_{2}\right)$ can be estimated in terms of herdisc $\left(\mathcal{F}_{1}\right)$ and herdisc $\left(\mathcal{F}_{2}\right)$ for any two set systems $\mathcal{F}_{1}$ and $\mathcal{F}_{2}$ (on the same vertex set). Hoffman's example mentioned above shows that herdisc $\left(\mathcal{F}_{1} \cup \mathcal{F}_{2}\right)$ cannot be bounded by a function of herdisc $\left(\mathcal{F}_{1}\right)$ and herdisc $\left(\mathcal{F}_{2}\right)$ alone.

The next theorem shows that a good bound is possible if we also allow for a moderate dependence on $m$ and $n$. Namely, herdisc $\left(\mathcal{F}_{1} \cup \mathcal{F}_{2}\right)$ can exceed $\operatorname{herdisc}\left(\mathcal{F}_{1}\right)+$ herdisc $\left(\mathcal{F}_{2}\right)$ at most by a factor polylogarithmic in $n$ and $m$, not much more than in Hoffman's example 3

The only previous result in this direction, from [KMV05, shows that if $\mathcal{F}_{2}$ consists of a single set, then herdisc $\left(\mathcal{F}_{1} \cup \mathcal{F}_{2}\right)=O\left(\operatorname{herdisc}\left(\mathcal{F}_{1}\right) \log n\right)$.

Theorem 3. Let $\mathcal{F}$ be a system of $m$ sets on $n$ vertices, and let $\mathcal{F}=\mathcal{F}_{1} \cup \mathcal{F}_{2} \cup$ $\cdots \cup \mathcal{F}_{t}$. Then

$$
\operatorname{herdisc}(\mathcal{F}) \leq\left(\max _{i=1,2, \ldots, t} \operatorname{herdisc}\left(\mathcal{F}_{i}\right)\right) \cdot O(\sqrt{t} \log (m n) \sqrt{\log n}) .
$$

There exist systems $\mathcal{F}$ of $n$ sets on $n$ points with discrepancy of order $\sqrt{n}$ (e.g., systems derived from Hadamard matrices or random set systems; see, e.g., Mat10]). If we let $\mathcal{F}_{i}$ be the set system consisting of the $i$ th set of such an $n, i=1,2, \ldots, n$, then $\operatorname{herdisc}\left(\mathcal{F}_{i}\right)=1$, while herdisc $(\mathcal{F})=\Omega(\sqrt{n})$. In this sense, the bound in Theorem 3 is tight up to a polylogarithmic factor, including the dependence on $t$.

Theorem 3 is an immediate consequence of Theorems 11 and 2 and of the next linear-algebraic lemma.

Lemma 4. Let $A_{1}, \ldots, A_{t}$ be real matrices, each with $n$ columns; let $D:=$ $\max _{i=1,2, \ldots, t} \operatorname{detlb}\left(A_{i}\right)$; and let $A$ be a matrix in which each row is a row of some of the $A_{i}$. Then

$$
\operatorname{det} \operatorname{lb}(A) \leq D \sqrt{e t}
$$

\footnotetext{
${ }^{2}$ The vertex set in the example is the set of edges of the complete $k$-ary tree $T$ of depth $k$ (so $n \approx k^{k}$ ). The set system $\mathcal{F}$ is a union $\mathcal{F}_{1} \cup \mathcal{F}_{2}$, where $\mathcal{F}_{1}$ consists of the edge sets of all root-to-leaf paths, and $\mathcal{F}_{2}$ contains, for each non-leaf vertex $v$, the set of the $k$ edges connecting $v$ to its successors. We have herdisc $\left(\mathcal{F}_{1}\right) \leq 1, \operatorname{herdisc}\left(\mathcal{F}_{2}\right) \leq 1, \operatorname{detlb}(\mathcal{F})=O(1)$, and $\operatorname{disc}(\mathcal{F})=$ $k \approx(\log n) /(\log \log n)$. See, e.g., BS95] or [Mat10] for more details.

${ }^{3}$ A connection of Sós's question to the tightness of the determinant lower bound was observed in [LSV86], although with a different proof which would yield a quantitatively weaker result in our setting.
} 
In the case where some of the $\operatorname{detlb}\left(A_{i}\right)$ are much smaller than the others, for example, one can obtain a somewhat better upper bound by making finer estimates in the calculation in the proof. However, a general formulation of such a finer bound looks cumbersome, and it seems that in such cases, a similar improvement can usually be achieved by applying the lemma repeatedly in several stages with various values of $D$.

The bound in Lemma 4 is generally tight up to a constant factor; this can be seen from an example similar to the one mentioned below Theorem 3 . Namely, let $A$ be an $n \times n$ Hadamard matrix (i.e., a matrix with pairwise orthogonal rows and with \pm 1 entries, which is well known to exist for infinitely many values of $n$ ), and let $A_{i}$ be the single-row matrix made of the $i$ th row of $A, i=1,2, \ldots, n$. Then, obviously, $\operatorname{detlb}\left(A_{i}\right)=1$, while $\operatorname{detlb}(A) \geq \operatorname{det}(A)^{1 / n}=\sqrt{n}$. Moreover, if we partition the rows of this $A$ into $t$ blocks $A_{1}, \ldots, A_{t}$ by $n / t$ rows each, then the Hadamard bound, stating that the determinant of a matrix is at most the product of the Euclidean norms of the rows, implies $\operatorname{detlb}\left(A_{i}\right) \leq \sqrt{n / t}$. This shows the tightness of Lemma 4 for all $t \leq n$.

Vector discrepancy and Bansal's algorithm. The proof of Theorem 2 is based on a recent breakthrough - an algorithm of Bansal [Ban10]. The algorithm produces a low-discrepancy coloring of a given set system, using semidefinite programming and a clever randomized rounding strategy. To state the consequence of Bansal's work that we will use, we first introduce another notion of discrepancy.

The vector discrepancy of the set system $\mathcal{F}$, denoted by $\operatorname{vecdisc}(\mathcal{F})$, is the smallest $D \geq 0$ for which there exist unit vectors $\mathbf{u}_{1}, \ldots, \mathbf{u}_{n} \in \mathbb{R}^{n}$ such that

$$
\left\|\sum_{j \in F_{i}} \mathbf{u}_{j}\right\| \leq D, \quad i=1,2, \ldots, m,
$$

where $\|\cdot\|$ is the Euclidean norm. So, for vector discrepancy, one colors by unit vectors instead of \pm 1 's. Since a \pm 1 coloring can also be regarded as a vector coloring by the vectors $\mathbf{e}_{1}=(1,0, \ldots, 0)$ and $-\mathbf{e}_{1}$, we have $\operatorname{vecdisc}(\mathcal{F}) \leq \operatorname{disc}(\mathcal{F})$.

The hereditary vector discrepancy hervecdisc $(\mathcal{F})$ is the maximum vector discrepancy of a restriction of $\mathcal{F}$ to a subset $J \subseteq V$.

Bansal's algorithm yields the following.

Theorem 5 (Bansal [Ban10]). We have

$$
\operatorname{herdisc}(\mathcal{F})=O(\log (m n)) \cdot \operatorname{hervecdisc}(\mathcal{F})
$$

for all $\mathcal{F}$.

We conjecture that the claim of Theorem 5 actually holds with $\sqrt{\log (m n)}$ instead of $\log (m n)$. If true, this would yield a similar improvement in Theorem 2 and get close to an asymptotically optimal bound, at least assuming that $m$ is bounded by a polynomial in $n$.

\section{A DUAl Formulation of the VeCtor Discrepancy}

Lemma 6. We have $\operatorname{vecdisc}(\mathcal{F}) \geq D$ if and only if there are nonnegative reals $w_{1}, \ldots, w_{m}$ with $\sum_{i=1}^{m} w_{i} \leq 1$ and reals $z_{1}, \ldots, z_{n}$ with $\sum_{j=1}^{n} z_{j} \geq D^{2}$ such that for all $\mathbf{x} \in \mathbb{R}^{n}$,

$$
\sum_{i=1}^{m} w_{i}\left(\sum_{j \in F_{i}} x_{j}\right)^{2} \geq \sum_{j=1}^{n} z_{j} x_{j}^{2} .
$$


Proof. We will use the duality of semidefinite programming in a routine way. In the usual standard form, the primal semidefinite program is the following optimization problem $(\mathrm{P})$ :

maximize $C \bullet X$ subject to $A_{1} \bullet X=b_{1}, \ldots, A_{m} \bullet X=b_{m}, X \in \mathrm{PSD}_{n}$,

where $C, A_{1}, \ldots, A_{m}$ are symmetric real $n \times n$ matrices, $\mathbf{b}=\left(b_{1}, \ldots, b_{m}\right) \in \mathbb{R}^{m}, X$ is an unknown $n \times n$ matrix, $\mathrm{PSD}_{n}$ denotes the cone of all $n \times n$ positive semidefinite matrices, and $\bullet$ is the scalar product of matrices (given by $X \bullet Y=\sum_{i, j} x_{i j} y_{i j}$ ). Then the dual semidefinite program (D) is

$$
\text { minimize } \mathbf{b}^{T} \mathbf{y} \text { subject to } \mathbf{y} \in \mathbb{R}^{m}, \sum_{i=1}^{m} y_{i} A_{i}-C \in \operatorname{PSD}_{n} \text {. }
$$

The duality theorem asserts that if the maximum of $(\mathrm{P})$ is bounded and there exists a positive definite matrix $Q$ (as opposed to only positive semidefinite) satisfying all the constraints of $(\mathrm{P})$, then $(\mathrm{D})$ is feasible as well and its minimum equals the maximum of $(\mathrm{P})$ (this can be found, sometimes with slight variations, in several books and many surveys, such as VB96, GM12.

In our case, we start with the vector program defining vecdisc $(\mathcal{F})$, and we convert it to an equivalent semidefinite program of the form $(\mathrm{P})$. We introduce a variable $Q$, which is an $n \times n$ matrix with $q_{i j}=\mathbf{u}_{i}^{T} \mathbf{u}_{j}$ (the Gram matrix of the $\mathbf{u}_{j}$ ); as is well known, $Q$ is of this form for some vectors $\mathbf{u}_{1}, \ldots, \mathbf{u}_{n}$ exactly if $Q \in \operatorname{PSD}_{n}$, where $\mathrm{PSD}_{n}$ denotes the cone of $n \times n$ positive semidefinite matrices. We also introduce a nonnegative scalar variable $t$, which will play the role of $D^{2}$.

For the constraint $\left\|\sum_{j \in F_{i}} \mathbf{u}_{j}\right\|^{2} \leq D^{2}$, we expand the left-hand side to $\sum_{j, k \in F_{i}} \mathbf{u}_{j}^{T} \mathbf{u}_{k}$, and then it translates to

$$
\left(\mathbf{a}_{i} \mathbf{a}_{i}^{T}\right) \bullet Q \leq t
$$

where $\mathbf{a}_{i}$ is the $i$ th row of the incidence matrix $A$ of $\mathcal{F}$, regarded as an $n \times 1$ matrix (so $\mathbf{a}_{i} \mathbf{a}_{i}^{T}$ is an $n \times n$ matrix). The constraint $\left\|\mathbf{u}_{j}\right\|=1$ reads

$$
E_{j} \bullet Q=1,
$$

where $E_{j}$ is the matrix with 1 at position $(j, j)$ and 0 s elsewhere. Since the constraints $A_{i} \bullet X=b_{i}$ in $(\mathrm{P})$ are equalities, we introduce nonnegative slack variables $s_{1}, \ldots, s_{m}$ and replace (2) with $\mathbf{a}_{i} \mathbf{a}_{i}^{T} \bullet Q+s_{i}-t=0$. Finally, we combine the matrix variable $Q$ and the scalar variables $t, s_{1}, \ldots, s_{m}$ into a single positive semidefinite $(n+m+1) \times(n+m+1)$ matrix $X$, which has $Q$ at the upper left corner, $t, s_{1}, \ldots, s_{m}$ on the diagonal below $Q$, and 0 s elsewhere. We also need to add the equality constraints forcing these $0 \mathrm{~s}$ in $X$. Finally, the objective function to be maximized is set to $-t$.

It is clear that the resulting $(\mathrm{P})$ has a feasible solution $\tilde{X}$ that is positive definite (indeed, choose arbitrary linearly independent unit vectors $\mathbf{u}_{1}, \ldots, \mathbf{u}_{n}$ and a gigantic $t$ ) and that the maximum is bounded from above (by 0 for sure).

Then, somewhat laboriously but routinely, we set up the dual program (D) and then we simplify it to the form asserted in the lemma. We omit this part.

We will use the duality of semidefinite programming. Dualizing a semidefinite program is a routine procedure, but unfortunately, I am not aware of an explicit recipe for the general case in the literature. Rather than converting the relevant semidefinite program to a standard form, it seems more convenient to use a duality theorem for conic programing from Duffin [Duf56, which we now introduce. 
Let $V, W$ be real vector spaces (for our purposes we may assume that they are finite-dimensional), each with a scalar product, which we denote by $\langle\cdot, \cdot\rangle$ in both cases. Let $K \subseteq V$ and $L \subseteq W$ be closed convex cones 4 let $\mathbf{b} \in W$ and $\mathbf{c} \in V$ be vectors, and let $F: V \rightarrow W$ be a linear map. We consider the primal cone program (P)

$$
\text { maximize }\langle\mathbf{c}, \boldsymbol{\xi}\rangle \text { subject to } \mathbf{b}-F(\boldsymbol{\xi}) \in L, \boldsymbol{\xi} \in K
$$

and the dual cone program (D)

$$
\text { minimize }\langle\mathbf{b}, \boldsymbol{\omega}\rangle \text { subject to } F^{T}(\boldsymbol{\omega})-\mathbf{c} \in K^{*}, \boldsymbol{\omega} \in L^{*} \text {. }
$$

Here $F^{T}: W \rightarrow V$ denotes the adjoint of $F$; if we fix orthonormal bases in $V$ and $W$, then the matrix of $F^{T}$ is the transpose of the matrix of $F$. The duality theorem asserts that if the maximum in (P) is a finite number $\gamma$ and if the set of feasible solutions of $(\mathrm{P})$ has an interior point, then (D) is feasible as well and its minimum equals $\gamma$. (Since (P) and (D) are dual to one another, one can also interchange their role in the theorem.)

In our case, we start with a vector program defining $\operatorname{vecdisc}(\mathcal{F})$, namely,

minimize $t$ subject to $\left\|\sum_{j \in F_{i}} \mathbf{u}_{j}\right\|^{2} \leq t, i=1,2, \ldots, m,\left\|\mathbf{u}_{1}\right\|=\cdots=\left\|\mathbf{u}_{n}\right\|=1$,

and we convert it to an equivalent semidefinite program in a standard way. We introduce a variable $Q$, which is an $n \times n$ matrix with $q_{i j}=\mathbf{u}_{i}^{T} \mathbf{u}_{j}$ (the Gram matrix of the $\mathbf{u}_{j}$ ); as is well known, $Q$ is of this form for some vectors $\mathbf{u}_{1}, \ldots, \mathbf{u}_{n}$ exactly if $Q \in \mathrm{PSD}_{n}$, where $\mathrm{PSD}_{n}$ denotes the cone of $n \times n$ positive semidefinite matrices.

For the constraint $\left\|\sum_{j \in F_{i}} \mathbf{u}_{j}\right\|^{2} \leq t$, we expand the left-hand side to $\sum_{j, k \in F_{i}} \mathbf{u}_{j}^{T} \mathbf{u}_{k}$, and then it translates to

$$
t-\left(\mathbf{a}_{i} \mathbf{a}_{i}^{T}\right) \bullet Q \geq 0,
$$

where $\mathbf{a}_{i}$ is the $i$ th row of the incidence matrix $A$ of $\mathcal{F}$, regarded as an $n \times 1$ matrix (so $\mathbf{a}_{i} \mathbf{a}_{i}^{T}$ is an $n \times n$ matrix), and $\bullet$ denotes the scalar product of matrices (given by $\left.X \bullet Y=\sum_{i, j} x_{i j} y_{i j}\right)$. The constraint $\left\|\mathbf{u}_{j}\right\|=1$ then reads

$$
E_{j} \bullet Q=1,
$$

where $E_{j}$ is the matrix with 1 at position $(j, j)$ and 0 s elsewhere.

This semidefinite program can be regarded as the cone program $(\mathrm{P})$ with $V=$ $\mathrm{SYM}_{n} \oplus \mathbb{R}$ (where $\mathrm{SYM}_{n}$ denotes the vector space of all symmetric $n \times n$ matrices with the $\bullet$ scalar product), $W=\mathbb{R}^{m} \oplus \mathbb{R}^{n}, K=\mathrm{PSD}_{n} \oplus[0, \infty)$, and $L=[0, \infty)^{m} \oplus$ $\left\{\mathbf{0}_{n}\right\}$ (where $\mathbf{0}_{n}$ denotes the $n$-component vector of $0 \mathrm{~s}$ ). We write the unknown $\boldsymbol{\xi} \in V$ in the form $(Q, t)$; then we have $F(Q, t)=\left(\mathbf{a}_{1} \mathbf{a}_{1}^{T} \bullet Q-t, \ldots, \mathbf{a}_{m} \mathbf{a}_{m}^{T} \bullet Q-\right.$ $\left.t, E_{1} \bullet Q, \ldots, E_{n} \bullet Q\right)$. Finally, $\mathbf{c}=\left(0_{n \times n},-1\right)$ and $\mathbf{b}=\left(\mathbf{0}_{m}, \mathbf{1}_{n}\right)$.

As for the dual cone program, it is well known that $\mathrm{PSD}_{n}^{*}=\mathrm{PSD}_{n}$, so $K^{*}=K$, and it is easily checked that $L^{*}=[0, \infty)^{m} \oplus \mathbb{R}^{n}$. Let us write the variable $\boldsymbol{\omega}$ in (D) in the form $(\mathbf{w},-\mathbf{z}), \mathbf{w} \in \mathbb{R}^{m}, \mathbf{z} \in \mathbb{R}^{n}$. Then (D) becomes

$$
\text { maximize } \sum_{j=1}^{n} z_{j} \text { subject to } \begin{aligned}
& \sum_{i=1}^{m} w_{i} \mathbf{a}_{i} \mathbf{a}_{i}^{T}-\sum_{j=1}^{n} z_{j} E_{j} \in \mathrm{PSD}_{n} \\
& 1-\sum_{i=1}^{m} w_{j} \geq 0, w_{1}, \ldots, w_{m} \geq 0
\end{aligned}
$$

${ }^{4} \mathrm{~A}$ convex cone is a convex set $K$ such that $\mathbf{x} \in K$ implies $\lambda \mathbf{x} \in K$ for all $\lambda \geq 0$. The dual cone of $K$ is $K^{*}=\{\mathbf{y}:\langle\mathbf{x}, \mathbf{y}\rangle \geq 0$ for all $\mathbf{x} \in K\}$. A simple property we will often use is $(K \oplus L)^{*}=K^{*} \oplus L^{*}$, where $\oplus$ denotes direct sum; we assume $K \subseteq V, L \subseteq W$, where $V, W$ are disjoint vector spaces, and $K \oplus L \subseteq V \oplus W$. 
Let $M$ stand for the matrix $\sum_{i=1}^{m} w_{i} \mathbf{a}_{i} \mathbf{a}_{i}^{T}-\sum_{j=1}^{n} z_{j} E_{j}$. Then the condition of positive semidefiniteness of $M$ means that $\mathbf{x}^{T} M \mathbf{x} \geq 0$ for all $\mathbf{x} \in \mathbb{R}^{n}$, and it is easy to verify that this can be rewritten as the inequality (1) in the lemma.

It remains to verify that the duality theorem can be applied to these $(\mathrm{P})$ and (D). We will check that (D) is bounded and has a feasible interior point. To verify boundedness, which means that $\sum_{j=1}^{n} z_{j}$ cannot be arbitrarily large, we use (11) with $\mathbf{x}=\mathbf{1}_{n}$ : then the left-hand side is bounded, and so $\sum_{j=1}^{n} z_{j}$ is bounded as well.

For an interior feasible point, we can take, e.g., $w_{1}=\cdots=w_{m}=\frac{1}{2 m}, z_{1}=\cdots=$ $z_{n}=-1$. Then (1) obviously holds for all $\mathbf{x}$.

Thus, the duality theorem applies and shows that if $\operatorname{vec} \operatorname{disc}(\mathcal{F}) \geq D$, then the maximum in (6) is at least $D^{2}$. This yields the existence of the desired $w_{i}$ and $z_{j}$, and the lemma is proved.

\section{Proof of Theorem 2}

We begin with a simple and probably standard lemma.

Lemma 7. Let $\mathbf{y} \in \mathbb{R}^{n}$ be a vector. Then there exists a subset $K \subseteq[n]$ of indices such that $\mathbf{y}$ is "almost constant" on $K$, in the sense that, for some $t>0$, we have $t<\left|y_{j}\right| \leq 2 t$ for all $j \in K$, and

$$
\|\mathbf{y}[K]\| \geq \Omega\left(\frac{\|\mathbf{y}\|}{\sqrt{\log n}}\right),
$$

where $\mathbf{y}[K]$ denotes the $|K|$-component vector $\left(y_{j}: j \in K\right)$.

Proof. Let $y_{\max }:=\max _{j}\left|y_{j}\right|$, and for $i=0,1,2, \ldots$, let

$$
K_{i}:=\left\{j:\left|y_{j}\right| \in\left(2^{-i-1} y_{\max }, 2^{-i} y_{\max }\right]\right\} .
$$

The contribution to $\|\mathbf{y}\|$ of the components of $\mathbf{y}$ with indices in $K_{i}$ for $i \geq 2 \log n$, say, is negligible, and so there exists some $i_{0}$ for which $\sum_{j \in K_{i_{0}}} y_{j}^{2}=\Omega\left(\|\mathbf{y}\|^{2} / \log n\right)$. Then $K:=K_{i_{0}}$ will do.

Theorem 2 will follow from Bansal's result (Theorem 5) and the next lemma.

Lemma 8. Let $\mathcal{F}=\left\{F_{1}, \ldots, F_{m}\right\}$ be a set system on $[n]$ with $\operatorname{vecdisc}(\mathcal{F})=D$. Then $\operatorname{det} \operatorname{lb}(\mathcal{F})=\Omega(D / \sqrt{\log n})$.

Proof. We begin with the dual formulation of vector discrepancy from Lemma 6, For more convenient notation, we will write the nonnegative weight $w_{i}$ as $\beta_{i}^{2}$. Moreover, we let $J \subseteq[n]$ consist of the indices $j$ with $z_{j}>0$, and we will use the inequality (11) in Lemma 6 only for vectors $\mathbf{x}$ that are zero outside $J$. Writing $z_{j}=\gamma_{j}^{2}$ for $j \in J$, we arrive at the inequality

$$
\sum_{i=1}^{m} \beta_{i}^{2}\left(\sum_{j \in F_{i} \cap J} x_{j}\right)^{2} \geq \sum_{j \in J}^{n} \gamma_{j}^{2} x_{j}^{2}
$$

for all $\mathbf{x} \in \mathbb{R}^{J}$, where $\|\boldsymbol{\beta}\| \leq 1$ and $\|\boldsymbol{\gamma}\| \geq D$.

Next, using Lemma 7 for $\mathbf{y}=\boldsymbol{\gamma}$, we select $K \subseteq J$ with $\|\gamma[K]\|=\Omega(D / \sqrt{\log n})$ and with $\gamma[K]$ almost constant (within a factor of 2). Setting $k:=|K|$ and $\tilde{D}:=\frac{1}{2}\|\gamma[K]\|$, the quadratic average of $\gamma_{j}$ over $j \in K$ equals $2 \tilde{D} / \sqrt{k}$, and so 
$\gamma_{j} \geq \tilde{D} / \sqrt{k}$ for all $j \in K$. Therefore, restricting (7) to vectors $\mathbf{x}$ with $x_{j}=0$ for $j \notin K$, we obtain

$$
\sum_{i=1}^{m} \beta_{i}^{2}\left(\sum_{j \in F_{i} \cap K} x_{j}\right)^{2} \geq \frac{\tilde{D}^{2}}{k} \sum_{j \in K} x_{j}^{2}
$$

for all $\mathbf{x} \in \mathbb{R}^{K}$.

Let $C:=A[*, K]$ be the $m \times k$ incidence matrix of the system $\left.\mathcal{F}\right|_{K}$ (consisting of the columns of $A$ whose indices belong to $K$ ), and let $\check{C}$ be the $m \times k$ matrix obtained from $C$ by multiplying the $i$ th row by $\beta_{i}$. Then (8) can be rewritten as

$$
\mathbf{x}^{T} \check{C}^{T} \check{C} \mathbf{x}=\|\check{C} \mathbf{x}\|^{2} \geq \frac{\tilde{D}^{2}}{k}\|\mathbf{x}\|
$$

for all $\mathbf{x} \in \mathbb{R}^{k}$.

This, by the usual variational characterization of eigenvalues, tells us that the smallest eigenvalue of the $k \times k$ matrix $\check{C}^{T} \check{C}$ satisfies $\lambda_{\min }\left(\check{C}^{T} \check{C}\right) \geq \tilde{D}^{2} / k$. Then, since the determinant is the product of eigenvalues, we also have $\operatorname{det}\left(\check{C}^{T} \check{C}\right) \geq$ $\left(\tilde{D}^{2} / k\right)^{k}$. The Binet-Cauchy formula then asserts that

$$
\operatorname{det}\left(\check{C}^{T} \check{C}\right)=\sum_{I} \operatorname{det}(\check{C}[I, *])^{2},
$$

where the summation is over all $k$-element subsets $I \subseteq[m]$ and $\check{C}[I, *]$ consists of the rows of $\check{C}$ whose indices lie in $I$.

We have $\operatorname{det}(\check{C}[I, *])=\operatorname{det}(C[I, *]) \prod_{i \in I} \beta_{i}$. Setting $M:=\max _{I}|\operatorname{det}(C[I, *])|$, we can rewrite the right-hand side of (19) and estimate it as follows:

$$
\begin{aligned}
\sum_{I} \operatorname{det}(\check{C}[I, *])^{2} & =\sum_{I} \operatorname{det}(C[I, *])^{2} \prod_{i \in I} \beta_{i}^{2} \\
& \leq M^{2} \sum_{I} \prod_{i \in I} \beta_{i}^{2} \\
& \leq M^{2} \frac{\left(\sum_{i=1}^{m} \beta_{i}^{2}\right)^{k}}{k !} \leq \frac{M^{2}}{k !}
\end{aligned}
$$

where the penultimate inequality follows because every term $\prod_{i \in I} \beta_{i}^{2}$ occurs $k$ ! times in the multinomial expansion of $\left(\beta_{1}^{2}+\cdots+\beta_{m}^{2}\right)^{k}$.

Letting $B:=C[I, *]$ for an $I$ maximizing $|\operatorname{det} C[I, *]|$, we thus have $\operatorname{det}(B)^{2} \geq$ $k ! \operatorname{det}\left(\check{C}^{T} \check{C}\right) \geq k !\left(\tilde{D}^{2} / k\right)^{k} \geq(k / e)^{k}\left(\tilde{D}^{2} / k\right)^{k}=\Omega(\tilde{D})^{2 k}=\Omega(D / \sqrt{\log n})^{2 k}$. So the $k \times k$ matrix $B$ witnesses $\operatorname{det} \operatorname{lb}(\mathcal{F})=\Omega(D / \sqrt{\log n})$, and the lemma is proved.

Proof of Theorem 2. By Theorem 5, there is a subset $J \subseteq[n] \operatorname{with} \operatorname{vecdisc}\left(\left.\mathcal{F}\right|_{J}\right)=$ $\Omega(\operatorname{herdisc}(\mathcal{F}) / \log (m n))$. Theorem 2 follows by applying Lemma 8 to $\left.\mathcal{F}\right|_{J}$.

\section{Proof of Lemma 4}

Let us consider a $k \times k$ submatrix $B$ of the matrix $A$, and let $I_{1}, \ldots, I_{t}$ be index sets partitioning $[k]$ such that $B\left[I_{\ell}, *\right]$ is a submatrix of $A_{\ell}, \ell=1,2, \ldots, t$ (we also admit $\left.I_{\ell}=\emptyset\right)$. Let $k_{\ell}:=\left|I_{\ell}\right|$.

We now apply the Gram-Schmidt orthogonalization to the row vectors of each $B\left[I_{\ell}, *\right]$, separately for each $\ell$. We obtain a new matrix $\tilde{B}$ such that the rows of each $\tilde{B}\left[I_{\ell}, *\right]$ are orthogonal vectors. Let $\tilde{\mathbf{b}}_{i}$ be the $i$ th row of $\tilde{B}$. 
Since the orthogonalization proceeds by elementary row operations, which do not change the determinant, we have $\operatorname{det} \tilde{B}=\operatorname{det} B$. We estimate $\operatorname{det} B$ using the Hadamard bound

$$
|\operatorname{det} B|=|\operatorname{det} \tilde{B}| \leq \prod_{i=1}^{k}\left\|\tilde{\mathbf{b}}_{i}\right\|=\prod_{\ell=1}^{t} \prod_{i \in I_{\ell}}\left\|\tilde{\mathbf{b}}_{i}\right\| .
$$

Let us fix $\ell$ for a moment. In order to estimate $\prod_{i \in I_{\ell}}\left\|\tilde{\mathbf{b}}_{i}\right\|$, we consider the quantity $\operatorname{det}\left(\tilde{B}\left[I_{\ell}, *\right] \tilde{B}\left[I_{\ell}, *\right]^{T}\right)$. On the one hand, since the $\tilde{\mathbf{b}}_{i}$ are mutually orthogonal for $i \in I_{\ell}$, the matrix $\tilde{B}\left[I_{\ell}, *\right] \tilde{B}\left[I_{\ell}, *\right]^{T}$ is diagonal with the entries $\left\|\tilde{\mathbf{b}}_{i}\right\|^{2}, i \in I_{\ell}$, on the diagonal, and so

$$
\operatorname{det}\left(\tilde{B}\left[I_{\ell}, *\right] \tilde{B}\left[I_{\ell}, *\right]^{T}\right)=\prod_{i \in I_{\ell}}\left\|\tilde{\mathbf{b}}_{i}\right\|^{2} .
$$

On the other hand, by the Binet-Cauchy formula, we have (12)

$$
\operatorname{det}\left(\tilde{B}\left[I_{\ell}, *\right] \tilde{B}\left[I_{\ell}, *\right]^{T}\right)=\sum_{J \subseteq[k],|J|=k_{\ell}} \operatorname{det}\left(\tilde{B}\left[I_{\ell}, J\right]\right)^{2}=\sum_{J \subseteq[k],|J|=k_{\ell}} \operatorname{det}\left(B\left[I_{\ell}, J\right]\right)^{2},
$$

the last equality holding again because $\tilde{B}\left[I_{\ell}, J\right]$ is obtained from $B\left[I_{\ell}, J\right]$ by elementary row operations which preserve the determinant.

Putting (10), (11), and (12) together, we arrive at

$$
\operatorname{det}(B)^{2} \leq \prod_{\ell=1}^{t} \sum_{J \subseteq[k],|J|=k_{\ell}} \operatorname{det}\left(B\left[I_{\ell}, J\right]\right)^{2} \leq \prod_{\ell=1}^{t}\left(\begin{array}{c}
k \\
k_{\ell}
\end{array}\right) \operatorname{detlb}\left(A_{\ell}\right)^{2 k_{\ell}} \leq D^{2 k} \prod_{\ell=1}^{t}\left(\begin{array}{c}
k \\
k_{\ell}
\end{array}\right),
$$

since each $B\left[I_{\ell}, J\right]$ is a $k_{\ell} \times k_{\ell}$ submatrix of $A_{\ell}$.

Then we estimate, using the concavity of the function $x \mapsto x \ln (1 / x)$ and Jensen's inequality,

$$
\begin{aligned}
\prod_{\ell=1}^{t}\left(\begin{array}{c}
k \\
k_{\ell}
\end{array}\right) & \leq \prod_{\ell=1}^{t}\left(\frac{e k}{k_{\ell}}\right)^{k_{\ell}}=e^{\sum_{\ell=1}^{t} k_{\ell} \ln \left(e k / k_{\ell}\right)} \\
& \leq e^{t(k / t) \ln (e t)}=(e t)^{k}
\end{aligned}
$$

Thus $\operatorname{det}(B)^{1 / k} \leq D \sqrt{e t}$, as claimed.

Remark. The case $t=2$ has a somewhat simpler proof using the Laplace expansion of $\operatorname{det} B$, which asserts that

$$
\operatorname{det} B=\sum_{J} \operatorname{sgn}(I, J) \operatorname{det}(B[I, J]) \operatorname{det}(B[\bar{I}, \bar{J}]),
$$

where the sum is over all $|I|$-element subsets $J \subseteq[k], \bar{I}=[k] \backslash I$, and $\operatorname{sgn}(I, J) \in$ $\{ \pm 1\}$ is a sign depending on $I$ and $J$ in a way that is of no concern for us (see, e.g., [BJN83, Theorem 4.3]).

In our case, we again let $I_{\ell}$ be the set of indices of the rows of $B$ that come from $A_{\ell}, \ell=1,2$, and $k_{\ell}=\left|I_{\ell}\right|$. We use the Laplace expansion of $\operatorname{det} B$ for $I=I_{1}$. For every $J$ we have $\left|\operatorname{det}\left(B\left[I_{1}, J\right]\right)\right| \leq D^{k_{1}}$ and $\left|\operatorname{det}\left(B\left[I_{2}, \bar{J}\right]\right)\right| \leq D^{k_{2}}$ by the assumption, and so $\operatorname{det} B \leq\left(\begin{array}{c}k \\ k_{1}\end{array}\right) D^{k} \leq 2^{k} D^{k}$. Hence $\operatorname{det}(B)^{1 / k} \leq 2 D$, as needed.

A bound on $\operatorname{detlb}(A)$ for larger $t$ can also be obtained by iterating this argument, but this method apparently leads only to $\operatorname{detlb}(A)=O(t D)$. 


\section{PÁlVÖlgYI'S EXAMPLE}

As was pointed out by Pálvölgyi (private communication, 2011), his geometric construction in Pál10 actually provides a slight quantitative improvement over Hoffman's example. Translated to the setting of set systems, the construction yields, for every $k \geq 1$, two systems $\mathcal{F}_{1}, \mathcal{F}_{2}$ of $k$-element subsets of $[n]$, with $n=$ $\left(\begin{array}{c}2 k \\ k\end{array}\right)-1<4^{k}$, such that

(i) $\operatorname{herdisc}\left(\mathcal{F}_{1}\right)$, herdisc $\left(\mathcal{F}_{2}\right) \leq 1$, and

(ii) under every two-coloring of $[n], \mathcal{F}_{1} \cup \mathcal{F}_{2}$ contains a monochromatic set (and consequently, $\left.\operatorname{disc}\left(\mathcal{F}_{1} \cup \mathcal{F}_{2}\right)=k\right)$.

Since the construction in Pál10 is presented geometrically, and property (i) is not entirely obvious, we provide a short self-contained exposition.

The construction is inductive, and it requires two parameters, $k$ and $\ell$. The inductive hypothesis is the following:

For every $k, \ell \geq 1$ and a ground set $V$ of $n=\left(\begin{array}{c}k+\ell \\ k\end{array}\right)-1$ elements, there exist set systems $\mathcal{F}_{1}=\mathcal{F}_{1}(V, k, \ell), \mathcal{F}_{2}=\mathcal{F}_{2}(V, k, \ell)$ on $V$ such that $\mathcal{F}_{1}$ consists of $k$-tuples, $\mathcal{F}_{2}$ consists of $\ell$-tuples, herdisc $\left(\mathcal{F}_{1}\right)$, herdisc $\left(\mathcal{F}_{2}\right) \leq 1$, and for every red-blue coloring of $V$, there exists a set $F \in \mathcal{F}_{1}$ that is completely red or a set $F \in \mathcal{F}_{2}$ that is completely blue.

For $k=1$ and $\ell$ arbitrary, we have $n=\ell$, and we take $\mathcal{F}_{1}$ consisting of the $\ell$ singleton subsets of $V$, while $\mathcal{F}_{2}=\{V\}$. The other base case $\ell=1$ and $k$ arbitrary is symmetric.

In the inductive step, we take the ground set $V$ and decompose it into three disjoint subsets: $V^{\prime}$ with $\left(\begin{array}{c}k+\ell-1 \\ k-1\end{array}\right)-1$ elements, $V^{\prime \prime}$ with $\left(\begin{array}{c}k+\ell-1 \\ k\end{array}\right)-1$ elements, and a singleton set $\{p\}$. We inductively construct $\mathcal{F}_{1}^{\prime}:=\mathcal{F}_{1}\left(V^{\prime}, k-1, \ell\right)$ and $\mathcal{F}_{2}^{\prime}:=\mathcal{F}_{2}\left(V^{\prime}, k-1, \ell\right)$, as well as $\mathcal{F}_{1}^{\prime \prime}:=\mathcal{F}_{1}\left(V^{\prime \prime}, k, \ell-1\right)$ and $\mathcal{F}_{2}^{\prime \prime}:=\mathcal{F}_{2}\left(V^{\prime \prime}, k, \ell-1\right)$. Then we set $\mathcal{F}_{1}:=\left\{F \cup\{p\}: F \in \mathcal{F}_{1}^{\prime}\right\} \cup \mathcal{F}_{1}^{\prime \prime}, \mathcal{F}_{2}:=\mathcal{F}_{2}^{\prime} \cup\left\{F \cup\{p\}: F \in \mathcal{F}_{2}^{\prime \prime}\right\}$, and check the required properties by a straightforward induction.

We begin with verifying that under every red-blue coloring of $V, \mathcal{F}_{1}$ has a completely red set or $\mathcal{F}_{2}$ has a completely blue set. If $p$ is red, then we restrict the coloring on $V^{\prime}$ and inductively find a red $(k-1)$-tuple in $\mathcal{F}_{1}^{\prime}$, which together with $p$ gives a red $k$-tuple in $\mathcal{F}_{1}$, or a blue $\ell$-tuple in $\mathcal{F}_{2}^{\prime}$, which is also a blue $\ell$-tuple in $\mathcal{F}_{2}$. The case of $p$ blue is analogous.

It remains to verify that herdisc $\left(\mathcal{F}_{1}\right), \operatorname{herdisc}\left(\mathcal{F}_{2}\right) \leq 1$. By symmetry of the construction, it suffices to check herdisc $\left(\mathcal{F}_{1}\right)$. We inductively prove a slightly stronger statement: for every $W \subseteq V$, there is a \pm 1 coloring $\chi$ of $W$ such that $\chi(F \cap W) \in\{0,1\}$ for every $F \in \mathcal{F}_{1}$. The base cases with $k=1$ or $\ell=1$ are obvious, and in the inductive step, we inductively color $W^{\prime}=V^{\prime} \cap W$ and $W^{\prime \prime}=V^{\prime \prime} \cap W$, we flip the colors on $W^{\prime}$, and we color $p$ with +1 .

\section{ACKNowledGments}

The author would like to thank Nikhil Bansal for enlightening e-mail discussions concerning his algorithm and for help with dualizing the semidefinite program, and Dömötör Pálvölgyi for explaining to the author how his construction in Pál10] improves on Hoffmann's example. 


\section{REFERENCES}

[Ban10] N. Bansal. Constructive algorithms for discrepancy minimization. http://arxiv.org/ abs/1002.2259, also in FOCS'10: Proc. 51st IEEE Symposium on Foundations of Computer Science, pages 3-10, 2010.

[BJN83] P. B. Bhattacharya, S. K. Jain, and S. R. Nagpaul. First course in linear algebra. Wiley Eastern Limited, New Delhi, 1983. MR719018 (84m:15001)

[BS95] J. Beck and V. Sós. Discrepancy theory. In Handbook of Combinatorics, pages 14051446. North-Holland, Amsterdam, 1995. MR:1373682 (96m:11060)

[Duf56] R. J. Duffin. Infinite programs. In H. W. Kuhn and A. W. Tucker, editors, Linear Inequalities and Related Systems, volume 38 of Annals of Mathematical Studies, pages 157-170, 1956. MR0087573 (19:374a)

[GM12] B. Gärtner and J. Matoušek, Approximation algorithms and semidefinite programming. Springer, Heidelberg, 2012.

[KMV05] J.-H. Kim, J. Matoušek, and V.H. Vu. Discrepancy after adding a single set. Combinatorica, 25:499-501, 2005. MR2143253 (2006d:05180)

[LSV86] L. Lovász, J. Spencer, and K. Vesztergombi. Discrepancy of set-systems and matrices. European J. Combin., 7:151-160, 1986. MR856328 (88b:05036)

[Mat10] J. Matoušek. Geometric Discrepancy (An Illustrated Guide), 2nd printing. SpringerVerlag, Berlin, 2010. MR2683232 (2011h:11081)

[Pál10] D. Pálvölgyi. Indecomposable coverings with concave polygons. Discrete Comput. Geom., 44(3):577-588, 2010. MR2679054 (2011f:52008)

[VB96] L. Vandenberghe and S. Boyd. Semidefinite programming. SIAM Rev., 38:49-95, 1996. MR1379041 (96m:90005)

Department of Applied Mathematics and Institute of Theoretical Computer Science (ITi), Charles University, Malostranské nám. 25, 11800 Praha 1, Czech Republic 